\title{
Histogram Equalized Thresholding method for analysis of Diabetic Myonecrosis related images
}

\author{
${ }^{* 1}$ Fahimuddin Shaik, ${ }^{2}$ Gautam Bommagani \\ ${ }^{* 1}$ Department of Electronics and Communication Engineering, \\ Annamacharya Institute of Technology \& Science, Rajampeta, \\ Telangana, India \\ fahimaits@gmail.com \\ ${ }^{2}$ University of Houston, 4800 Calhoun Rd, Houston, TX, United States \\ gbommagani@uh.edu
}

Received: $23^{\text {rd }}$ August 2021, Accepted: $28^{\text {th }}$ September 2021, Published: $30^{\text {th }}$ October 2021

\begin{abstract}
Diabetes Mellitus has become a common disorder in all groups of people throughout the world in the current days, mainly because of sedentary lifestyles and abnormal eating habits. Moreover, it has become a threat as well as a challenge to the researchers in the scientific society to find a solution to control this problem. Even though there is no proven cure for this disorder, one can lead a normal life with knowledge and awareness of issues related to diabetes. The main issue of concern for people with diabetes is its related complications, especially Myonecrosis. Diabetic myonecrosis is a rare complication of diabetes mellitus that has been poorly addressed. The disease presents itself in a human as lower extremity acute non-traumatic swelling and pain that can imitate deep vein thrombosis (DVT). Usually, the medical condition is self-limiting, and patients react well to therapeutic treatment that supports them. Together with other microvascular complications such as retinopathy, nephropathy, or neuropathy, it often develops into further complications. These complications are more common among Type I diabetics but can also arise in patients with Type II diabetes. Current image processing methodologies hold a significant position in removing specific challenges related to medical imaging technologies. The research work dealt with in this paper is about the processing of images related to Diabetic Myonecrosis, and the outcome results are used for analysis of the problem in various conditions of DM patient with Myonecrosis. Threshold segmentation methods are used to extract the features, and several attributes have been derived for statistical comparison in this work. The tools used for investigation in this work are MATLAB Technical computing language for simulation results and MIPAV for deriving statistical parameters.
\end{abstract}

Keywords: Diabetes Mellitus; Diabetic Myonecrosis; Disorder; Enhancement; Thresholding.

\section{Introduction}

Diabetes Mellitus (DM)

DM is considered to be a very significant human health threat, as well as a substantial socio-economic, weigh-down on governments. According to statistics from the International Diabetes Federation (IDF), the approximate global occurrence of DM had reached 8.8\% in 2015 and 12\% of global health spending in the same year was to address DM. Diabetic Mellitus (DM) is a metabolic disorder that does not control the concentration of blood glucose in the pancreas. The ailment can lead to blood glucose levels being out of range [2]. In other words, Diabetes mellitus can be described as a persistent ailment that is caused by an innate and acquired dearth in the production of insulin by the pancreas, or by the futility of the insulin formed. Such a shortage results in increased concentrations of glucose in the blood, which in turn harm different part of the body's systems, by stressing out the blood vessels and nerves. 
There are two standard types of diabetes as per the categorization process:

- Type 1 diabetes (previously identified as insulin-dependent): The insulin that is necessary for life sustenance is due to the inability of the pancreas to generate the same. This form is evident most commonly among children and adults, and is alarmingly emphasized later in life.

- Type 2 diabetes (previously known to be non-insulin-dependent): This occurs mostly from the lack of ability of the body to respond appropriately to the pancreas generated insulin.

\section{Complications related to Diabetes Mellitus}

Type 2 diabetes is much more common and accounts for around 90\% of all diabetes cases globally. It happens for the most part regularly in adults but is being observed progressively more in teenagers as well. The most common complications of DM are Diabetic Retinopathy, Diabetic Nephropathy, Diabetic Neuropathy, and Diabetic Cardiomyopathy. Diabetic Retinopathy is one of the most common complications of diabetes. In people with Type 1 and Type 2 diabetes, Diabetic Retinopathy usually affects both eyes. For people with retinopathy, the blood vessels in the light-sensitive layer at the back of the eyes get damaged. Diabetic Retinopathy develops slowly and continues to evolve as time progresses over many years. The reduced supply of blood to the retina in the advanced stage of this ailment stimulates the growth of new blood vessels that grow across the retina and into the middle of the eyes. These are abnormal and extremely fragile blood vessels. They leak fluid and bleed, which can cause scar tissue to form.

Diabetic Nephropathy refers to kidney diseases occurring in individuals with diabetes. The kidneys help maintain the body's quantity of fluids and salts, which helps to regulate blood pressure and releases various hormone types. Nephropathy is the word used when the kidneys begin to cause serious harm, which can eventually lead to failure of the kidneys.

Diabetic Neuropathy is a severe and prevalent Type 1 and Type 2 diabetes complication. It is a form of nerve damage triggered by long term high levels of blood sugar. The disorder develops slowly over several decades. Diabetic Neuropathy has been classified into different types that affect different areas of the body, causing a variety of symptoms. Symptoms vary depending on the areas affected. Peripheral Neuropathy is the most frequent form of neuropathy. Usually, peripheral neuropathy affects the feet and legs, but it can also affect the arms or hands. Symptoms vary, and the severity may vary from mild to severe.

Diabetic Cardiomyopathy in people with diabetes is a heart muscle disorder. It may result in the lack of ability of the heart to effectively distribute blood through the body, a disorder called as heart failure, with accumulation of fluid in the lungs (pulmonary edema) or legs (peripheral edema). Moreover, most cardiac arrest is caused by coronary artery disease in diabetes patients, and Diabetic Cardiomyopathy occurs only if there is no cardiovascular disease and suggests that diabetes has produced damage to cardiac muscle.

\section{Diabetes Myonecrosis}

Diabetes mellitus is associated with microvascular and macrovascular complications; the most commonly recognized ones include diabetic nephropathy, retinopathy, cardiomyopathy, and neuropathy. Less wellknown complications such as myonecrosis are equally important, as timely recognition and treatment are essential to decrease short- and long-term morbidity. In long-term diabetes, a prominent issue of Myonecrosis with the patients may be prevalant. Even though it was first detailed in 1965, there have been only a few reporting and general review of these cases. [3,4] Clinical presentations involve acute muscle pain, usually in the thigh region. By far the most prevalently impacted muscle group is the thigh muscles (71.2 percent), such as quadriceps, hip adductors, hamstrings, and hip flexors. The second most common area involves calf muscles (15.3 percent). Diabetes Myonecrosis (also known as DMI-Diabetic Muscle Infarction) may be misdiagnosed as cellulite, thrombosis of deep veins or fasciitis. In the appropriate clinical context, magnetic resonance imaging (MRI) is sufficient to allow diagnosis, thus avoiding inappropriate treatment [5]. Severe pain is very typical of DMI and cannot be seen with fasciitis, or pyomyositis [7]. Diabetic myonecrosis pathophysiology is not fully understood; however, several theories 
have been put forward, including arteriosclerosis, microangiopathy, ischemia-reperfusion injury, and coagulation-fibrinolysis system alteration.

The choice test for investigation of such cases is MRI involving the muscle group, which is highly sensitive in the diagnosis of this disease, with and without contrast. Typical MRI findings include loss of intermuscular septae (most easily seen on T1-weighted images) and diffuse muscle enlargement with subcutaneous edema (most visualized on T2-weighted images as muscle hyperintensity compared to nonaffected muscle and fat). MRI images show diffuse muscle enhancement, which represents edema and inflammation, and a low-intensity signal focal area that suggests muscle necrosis or infarction. Even though muscle biopsy is the standard criterion for diagnosing diabetic myonecrosis, it is reserved for atypical patients when there are no suggestive clinical or radiological findings due to poor wound healing and high risk of muscle biopsy-related infection. The preferred first choice of investigation in such cases is medical imaging modality.

\section{Overview of Image Processing}

Image processing is a process of transforming an image into a digital form in order to derive an enhanced image or obtain useful data from it. It is a form of signal processing in which the input is an image, which may include a video frame or photograph, and output can be attributed based on the image. Generally, the Image Processing System provides classifying images as two-dimensional signals while submitting techniques of signal processing that have already been defined for them. Image processing is used on a practical note to extract significant features from the images, which allow human viewers to perceive the scene better [1]. The biological computing vision system is one of the world's most important means of human investigation, making it easier to understand complex tasks [6]. Advancements in the Image processing concepts have made the perception of the images more easy using various tools.

There are a variety of methods that can be used for different applications in image processing, but enhancement and segmentations are considered to be the most appropriate methods for improving image details. Any single technique cannot be considered superior in the applications of image processing, but the test and error method can be used as a practical process to attain the perfect results. Image enhancement is the most basic requirement in the processing and analysis of digital images to improve the appearance of the image in terms of perception for human cognition [8]. Image enhancement is used as the preprocessing procedure and segmentation is primarily valuable in the categorization of objects and labeling of image extracted features for easy post-processing investigation. Image enhancement procedures are mainly carried out to remove or reduce the effect of any irregularities added in the image during the image acquisition process.

Among the enhancement techniques, the histogram technique is considered valid because of their inherent properties to adapt to the situations and applications in hand. This technique is widely used in daily photo enhancement, medical image analysis, remote-sensing imagery, microscopic imaging [9], and many other areas [10-14]. Histogram equalization (HE) [15] is most extensively utilized for contrast enhancement. A digital image histogram is a distribution of its discrete levels of intensity in the range [0, L-1]. Histogram equalization is a method of processing images to adjust the image contrast by changing the histogram's intensity distribution. This technique aims to give a linear trend to the image related cumulative probability function. The histogram equalization processing is based on the use of the cumulative probability function. In particular, a limited Adaptive Histogram Equalization (AHE) is selected for our paper as conventional histogram equalization calculates a global equalization, while an adaptive method calculates several histograms. Furthermore, the process is applied to separate segments of the image and uses them to restructure the image's lightness values. It is suitable to improve the local contrast and improve edge definitions in each image region. 
A variant of adaptive histogram equalization, contrast-limited adaptive histogram equalization (CLAHE) limits a negative property of AHE to magnify noise in comparatively similar areas of an image. Moreover, AHE's second drawback is "cumulation function," which is useful when applied to grayscale images, but CLAHE can be utilized equally on colored and grayscale images [16]. In the segmentation process, thresholding techniques are used to create binary images, as thresholding is the simplest method for doing this. Thresholding is classified into two categories; Global Thresholding and Local Thresholding depending on the various distributions of image attributes.

\section{Methodology}

\section{Block Diagram}

The suggested method to enhance and segment the medical image to achieve the desired results of the paper is highlighted in the block diagram in Fig 1. A database of images associated with diabetes mellitus is obtained from public image repositories and used as input images. The images that are being used at this point are MRI images related to Diabetes Myonecrosis and are usually in RGB color structure. The RGB images are processed using the MATLAB software, and the images are subjected to several algorithms to achieve better output. The RGB image is initially transformed into a grayscale format to avoid complex calculations

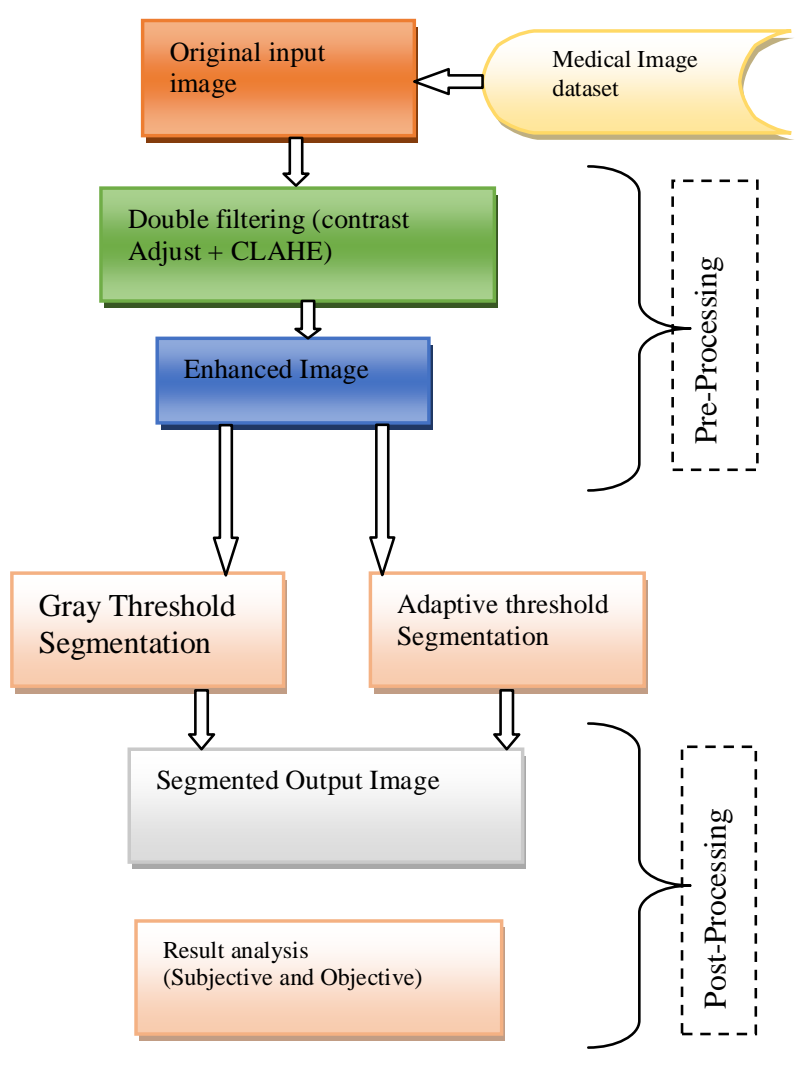

\section{Pre-Processing}

Fig 1: Implemented Model Block Diagram

In the pre-processing stage double filtering (contrast adjustment + Contrast Limited Adaptive Histogram Equalization) is done to enhance the medical images; both of the filtering (enhancement) methods belong to the family of Histogram Equalization. 
The first filtering process, referred to as contrast adjustment, is essential as images are short of contrast with no sharp differences between black and white can be viewed. Contrast adjustment remaps values of image intensity to the full data type display range. There are sharp diversities between black and white in an image with good contrast. Brightness refers to the overall lightness or darkness of an image. The Adjust Contrast tool carries out contrast stretching to change an image's contrast or brightness. In this process, black is mapped to pixel values lower than a particular value and white is mapped to pixel values exceeding a specified value. The consequence is a linear mapping of a subset of pixel values to the full range of display intensities. The process creates an image of advanced contrast by darkening pixels below a specified value and lightening pixels above a specified value. By tweaking this window over the display range, the Adjust Contrast tool adjusts the brightness without varying its size. The pixel values are thus mapped to lighter or darker intensities. The following figure 2 shows the mapping process as discussed above.

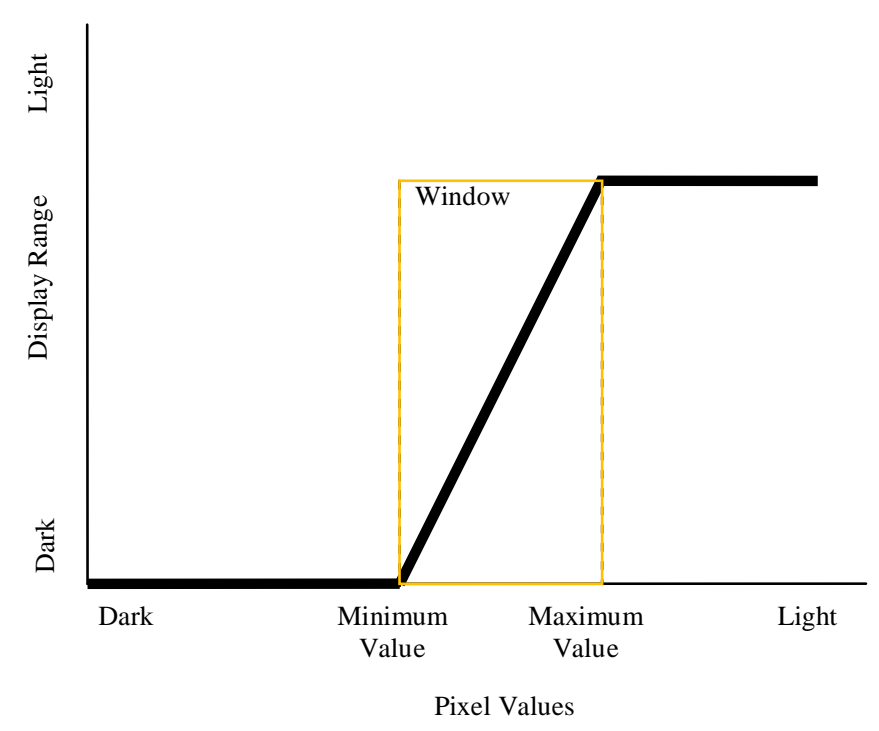

Fig 2: Mapping Process

A histogram is a graphical illustration of the intensity allocation of an image. In simple terms, it represents the number of pixels for every intensity value considered. Histogram Equalization is a processing technique used in computer image processing to improve image contrast.

The below equation can explain Histogram Equalization

$$
\mathrm{HE}=\text { No. of pixels with intensity } \mathrm{n}_{\mathrm{k}} / \text { Total no. of pixels } n
$$

Where $\mathrm{n}=0,1,2 \ldots \ldots . \mathrm{L}-1$, given as $[0 \ldots \mathrm{L}-1]$ is a range of the gray level values

Image contrast improvement is accomplished by disseminating the, for the most part, normal intensity values effectively, i.e., by extending the image intensity range. Usually, these processes increase the global contrast of images when secure contrast values represent their useable data. The process allows a higher contrast to areas with lower local contrast. This process is depicted in figure 3 where one can observe the distribution of pixels after the procedure. The filtering process has become inevitable in most of the medical imaging applications to remove noise elements added during the image acquisition through the imaging modalities. 


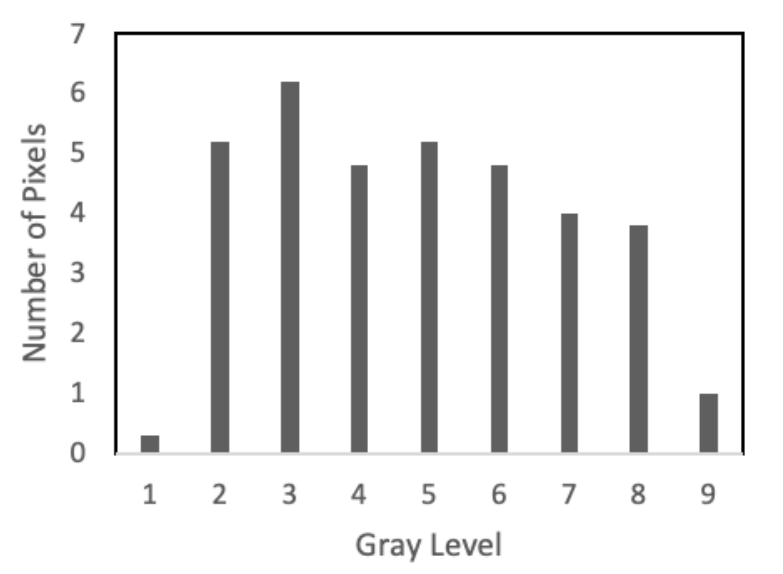

a)

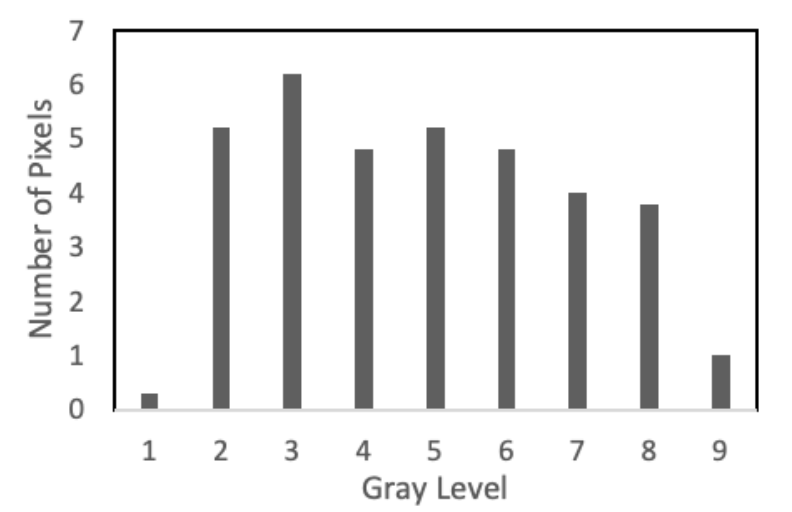

b)

Fig 3: Histogram values a) for original image b) for equalized image

The above graphs in figure 3 represent the number of pixels on the $y$-axis and grayscale values on the $\mathrm{x}$ axis. Contrast Limited Equalization of Adaptive Histogram (CLAHE) is projected to significantly decrease the over-amplification of noise issues present in the methodology of histogram equalization. It is fundamentally diverse from standardized histogram improvement. The process includes segregating and operating on small areas in the MRI image, called tiles and then further evaluating multiple histograms. All processed areas are put side by side to a specific area of the image and use them to reallocate the image's brightness or contrast estimation.

\section{Post-Processing}

In the post-processing stage, segmentation methods are employed to extract the final attributes that establish the significance of the application. In simple words, the purpose of segmentation is to differentiate one and sometimes more regions of interest in an image from regions that do not constitute relevant information. The segmentation of images is considered to be the first and fundamental procedure to evaluate and interpret the image acquired in numerous computer vision implementations such as decentralized target recognition, medical imaging, robotic vision, geographical imaging, [17]. It is frequently an essential stride in the direction of any additional processing and analysis [18-21]. Image segmentation is comprehensively applied in medical applications specified by its vital task in image processing, in treatment development monitoring, surgical planning, and abnormality recognition [22]. 
Medical imaging is a regulatory field within the medical field; this process involves a professional expert to restrict images of the internal parts of a human body being publicized [23].

The most convenient and commonly used method in segmentation is the thresholding method based on the image histogram [24]. The threshold should be automatically selected by the system to make segmentation more robust. Through thresholding, the separation of light and dark regions is conducted, a natural way to segment these regions. Hence the Gray thresholding (Global Thresholding using Otsu's method) and adaptive thresholding methods have been used to extract the features in the images under study.

Image thresholding is a straightforward nevertheless valuable approach to separate an image into the foreground and background values or parts. This technique of image analysis is a type of image segmentation with the aim of segregating objects by switching images of grayscale into a binary form of images.

$$
\mathrm{g}(\mathrm{x}, \mathrm{y})=\left\{\begin{array}{l}
\text { Foreground if } \mathrm{f}(\mathrm{x}, \mathrm{y}) \geq \mathrm{T} \\
\text { Background if } \mathrm{f}(\mathrm{x}, \mathrm{y})<\mathrm{T}
\end{array}\right.
$$

Local thresholding requires more effort than global thresholding to breakdown an image. In the specific case of images with diverse backgrounds, this mechanism is much more useful. Global thresholding is the fundamental technique of binarizing the image. A threshold limit is set in this response based on the histogram of the entire restored image or a set of images 25. Equation 2 does the representation for a single threshold value partitioning or bifurcation. It is also possible to have multiple thresholding bifurcations of histogram intensities which are represented in equation 3.

$$
\mathrm{g}(\mathrm{x}, \mathrm{y})=\left\{\begin{array}{c}
\mathrm{a}, \text { if } \mathrm{f}(\mathrm{x}, \mathrm{y})>T 2 \\
\mathrm{~b}, \text { if } \mathrm{T} 1<\mathrm{f}(\mathrm{x}, \mathrm{y})<T 2 \\
c, \text { if } f(x, y)<T 1
\end{array}\right.
$$

\subsubsection{Algorithm for Global Thresholding}

1. Initial estimate value of $\mathrm{T}$ $/ /(\mathrm{T}$ is referred to as Thresholding Value)

2. Image Segmentation using the Threshold value T:

- Referred to as G1 which are defined as pixels brighter than T;

- Referred to as G2 which are defined as pixels darker than or equal to T.

3. Calculation of the average intensity values denoted as $\mathrm{m} 1$ and $\mathrm{m} 2$ of $\mathrm{G} 1$ and $\mathrm{G} 2$ respectively.

4. Establishing a new threshold value given by $T_{\text {new }}=m 1+m 2 / 2$

5. If the condition $\left|T-T_{\text {new }}\right|>\Delta T$ arises, then loop back to step 2, or else end.

This process of thresholding is apparent from the following figure 4, Fig. 4 a) represents intensity histograms that are partitioned by a single threshold and 4 b) represents intensity histograms that are portioned by multiple thresholds (dual thresholds in this case).

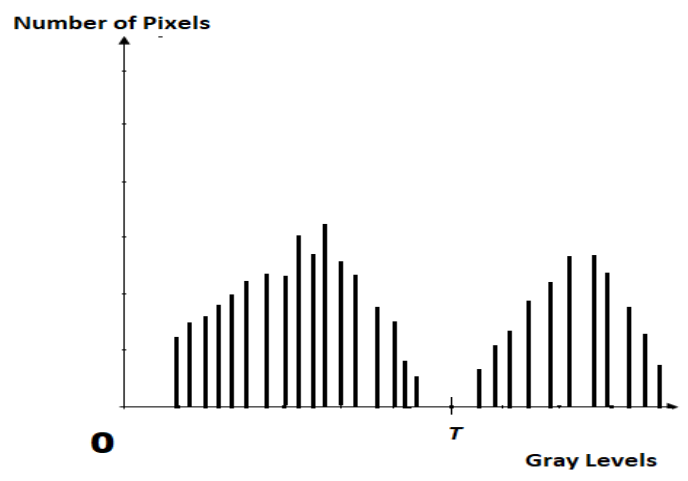

a) 


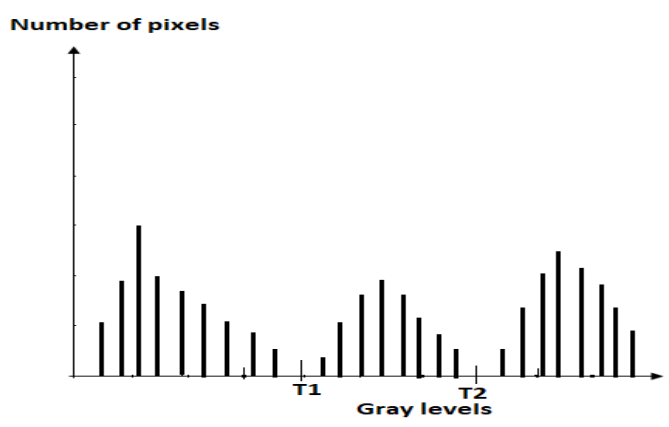

b)

Fig 4: Histogram partitioned by using a) single Threshold b) multiple Threshold

The properties of valleys shown in the figures 4 a) and b) are affected by parameters such as the separation between peaks, noise content, relative sizes of objects and background, uniformity of illumination source and uniformity of reflectance properties of the image.

In computer vision and image processing, the Otsu method, named after Nobuyuki Otsu, is used to carry out image thresholding using clustering or to reduce a grayscale image to a binary image. Otsu's technique is an incredibly straightforward histogram-based [26] thresholding approach designed for automatic image thresholding [27]. The method of Otsu selects the threshold by minimizing the variance within the class of the two-pixel groups separated by a particular thresholding operator as per the requirement. The algorithm tries to detect the best threshold value that separates the histogram into two subsections so that combined range is minimum or comparable "between class variance" is maximum [28] as depicted in figures 4 a) and 4 b). In this paper, the proposed double filtering with Gray Thresholding has given promising results.

Furthermore, the results presented in this paper are only of filtering with Gray Thresholding with Histogram process used for enhancement. Thus, the proposed method is named as Histogram Equalized Thresholding Method. A simple algorithm briefing the Otsu's method is given as simple steps in the following section.

\section{Simple process Algorithm for Otsu's method:}

1. Calculate normalized histogram of the selected image

2. Calculate cumulative sums of the intensities

3. Compute cumulative means

4. Compute the global intensity mean value

5. Compute between-class variance value

6. Attain the Otsu threshold value

7. Obtain the separability measure

\section{Pseudo Code of the entire process}

The entire implementation of this research can be written in the purest form of steps written as a pseudocode which is also referred to as an algorithm or process flow of the work.

Step 1: Start the code

Step 2: Load the image

Step 3: Read in the Color Image and Convert it to Grayscale

Step 4: Use the image adjust and image adaptive threshold as an enhancement Function

Step 5: Compute the gray threshold of the Segmentation Function using Otsu's method.

Step 6: Visualize the Result. 


\section{Results and Discussion}

\section{Existing Method-Results}

The original images used in this work are Coronal T1-weighted MRI images which have been acquired from a public repository (Image Courtesy: Orthopedics Journal, Healio [26] \& Applied Radiology Journal [27]) are shown in the figures 5 (a), 6(a) and 7 (a) in each condition of Diabetes Myonecrosis such as normal, medium and severe. The figures referred with (b), (c) and (d) represent single filtered images, double filtered images, and thresholder images respectively. The implementation is carried out using tool MATLAB with required toolboxes as per the application. The results presented in Fig 5. are of a person with Diabetes but with a controlled condition called as Normal Condition. The outcomes show that no traces of muscle infarction are present, which indicates no abnormalities. However, two other images of medium and severe conditions have shown some promising results in feature extraction, i.e., affected part of muscle represented in Fig 6 and Fig 7. As discussed earlier in the proposed method, double filtering has given the right amount of enhancement in the acquired images, helping us to get potential results shown as swelling of thigh muscle with arrow mark indicated as ROI (Region of Interest).

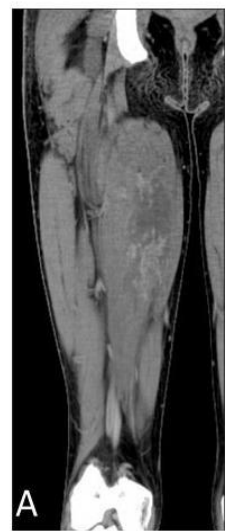

(a)

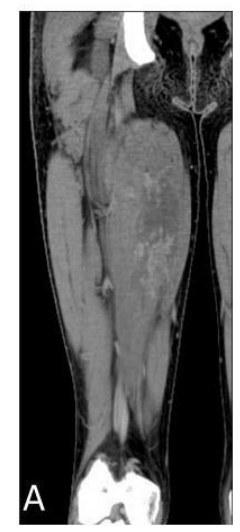

(b)

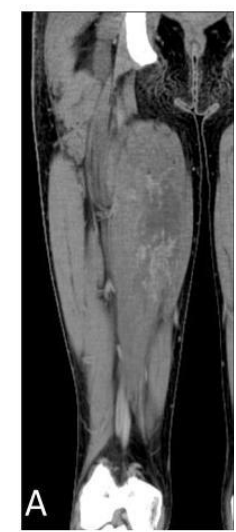

(c)

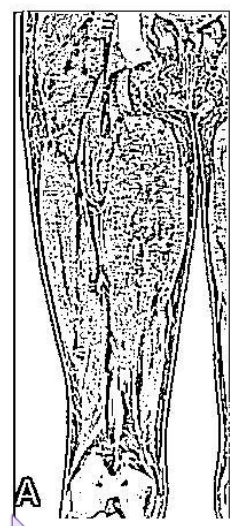

(d)

Fig 5: (a) Original Image (b) Single filtered Image (c) Double filtered Image (d) Threshold output Image

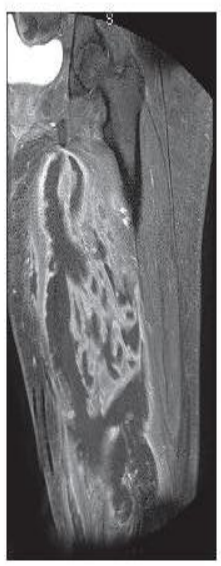

(a)

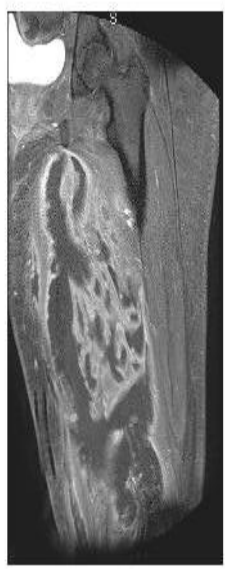

(b)

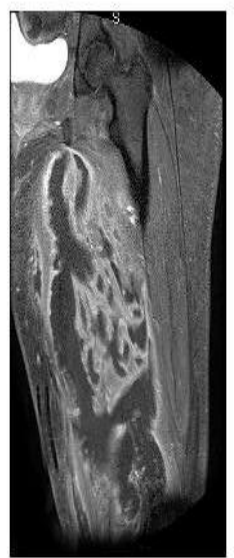

(c)

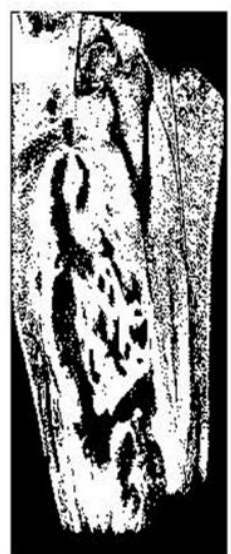

(d)

Fig 6: (a)

Original Image (b) Single filtered Image (c) Double filtered image (d) Threshold output 


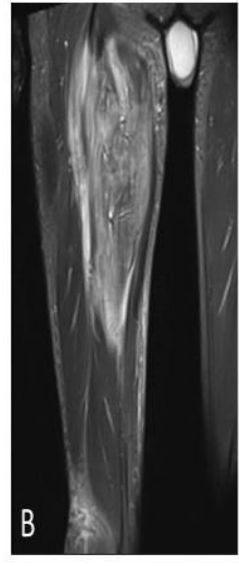

(a)

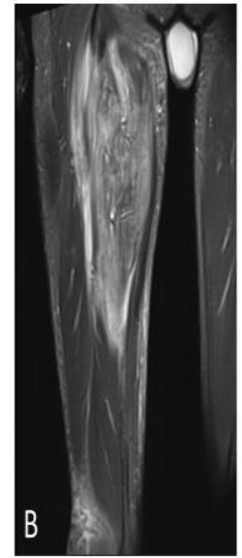

(b)

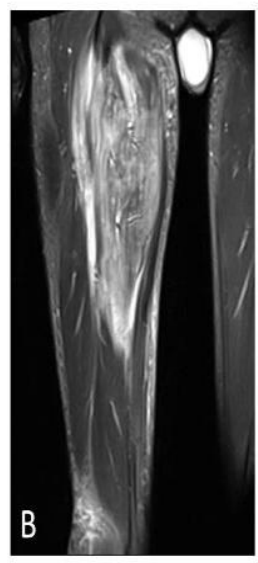

(c)

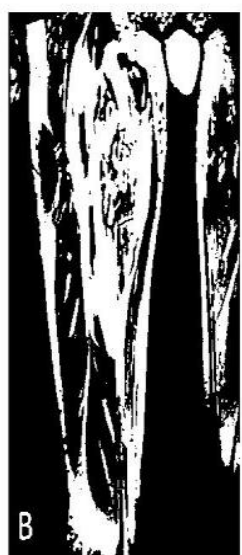

(d)

Fig 7: (a) Original Image (b) Single filtered Image (c) Double filtered image (d) Threshold output

\section{Statistical Analysis}

Even though the simulation results of existing methods have been not presented in this paper, a comparison between the methods has been presented in Table.1. The parameters are attained using the Medical Image Processing and Visualization (MIPAV) tool, which is an open source tool.

Table 1: Comparative analysis between existing and proposed methods

\begin{tabular}{|l|l|l|l|l|l|l|}
\hline Parameters & \multicolumn{2}{|c|}{ Normal condition } & \multicolumn{2}{c|}{ Medium condition } & \multicolumn{2}{c|}{ Severe condition } \\
\hline & \multicolumn{2}{|c|}{ Output image } & \multicolumn{2}{c|}{ Output image } & \multicolumn{2}{l|}{ Output image } \\
\cline { 2 - 7 } & Existing & Proposed & Existing & Proposed & Existing & Proposed \\
\hline Area & 7951 & 30070 & 15009 & 15009 & 25668 & 26351 \\
\hline Perimeter & 843.4373 & 714.4122 & 546.9645 & 546.9645 & 633.7572 & 780.2057 \\
\hline Circularity & 0.4937 & 0.7404 & 0.6304 & 0.6304 & 0.8031 & 0.544 \\
\hline $\begin{array}{l}\text { Std } \\
\text { deviation of } \\
\text { intensities }\end{array}$ & 114.244 & 105.3541 & 123.0383 & 124.0579 & 127.6062 & 130.3366 \\
\hline $\begin{array}{l}\text { Sum of } \\
\text { intensities }\end{array}$ & 4898994 & 5886346 & 2119017 & 2946234 & 4272492 & 4753726 \\
\hline \begin{tabular}{l} 
Median \\
\hline
\end{tabular} & 249 & 255 & 200 & 217 & 251 & 259 \\
\hline
\end{tabular}

Simple statistical descriptions of images and sub-images are quite common in image processing. The analysis of image statistical properties is dictated by the concern of adapting to the image signal subsequent treatments such as filtering, restoring, coding and form recognition. However, statistical representation is 
quite useful for automatic computer processing as it immediately feeds more or less elaborate algorithms that manipulate pixels, the essential components of the image.

An open source medical image processing, analysis and visualization (MIPAV) is used to extract meaningful attributes from the images. The parameters are productive in clarifying the abnormalities accurately. The main feature that makes MIPAV to be efficiently used by the researcher is its applicability on 3D images and the characteristic of quantification. MIPAV belongs to Java-based app family which can be implemented practically on any PC facilitated by the Java tool. This tool is a product of Center for Information Technology, National Institutes of Health, Bethesda, MD, USA.

When using the MIPAV tool, Region of Interest (ROI) is considered as Volumes of Interest (VOI) as mentioned in the results. Each VOI can be formed from multiple contours in one slice or multiple slices. Once a VOI is drawn, the different attributes tabulated in Table. 1 can be calculated. The computations are made only for the images of the output, and not for every image attained in the assessment. The parameters area and parameters are fundamental values which are calculated based on the number of pixels available in the particular VOI zone.

The median parameter is considered instead of the mean as a location parameter for obtaining proper thresholds due to its inherent property of robustness. In most of the applications, the median is used to estimate the dispersion instead of the variance. The median parameter is the result of an average number of pixels in the VOI (i.e., ROI).

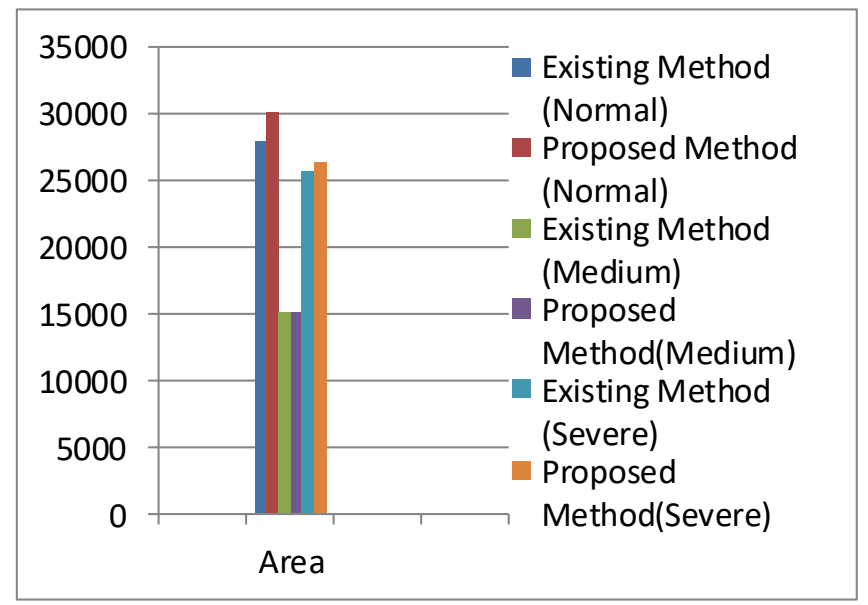

Fig 8: Graphical Representation of Area for existing and proposed methods

The standard deviation is similar to the average deviation, except that the average is calculated using power rather than amplitude. The standard deviation is inferred before taking the average by squaring each of the deviations. The standard deviation presents a quantity of the spreading of image grey level intensities and can be assumed as an amount of the power level of the alternating signal module obtained by the imaging modality. This parameter reflects the degree of deviation amid the whole image and the resulted mean image. In simple words, the standard deviation is an estimate of a function of the underlying brightness probability distribution. 


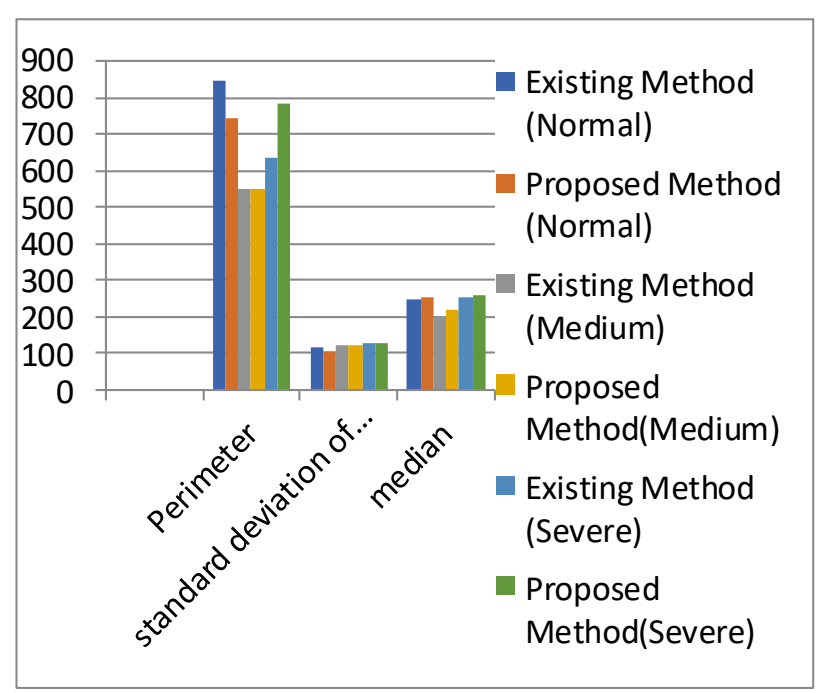

Fig 9: Median, Standard Deviation and Perimeter representation

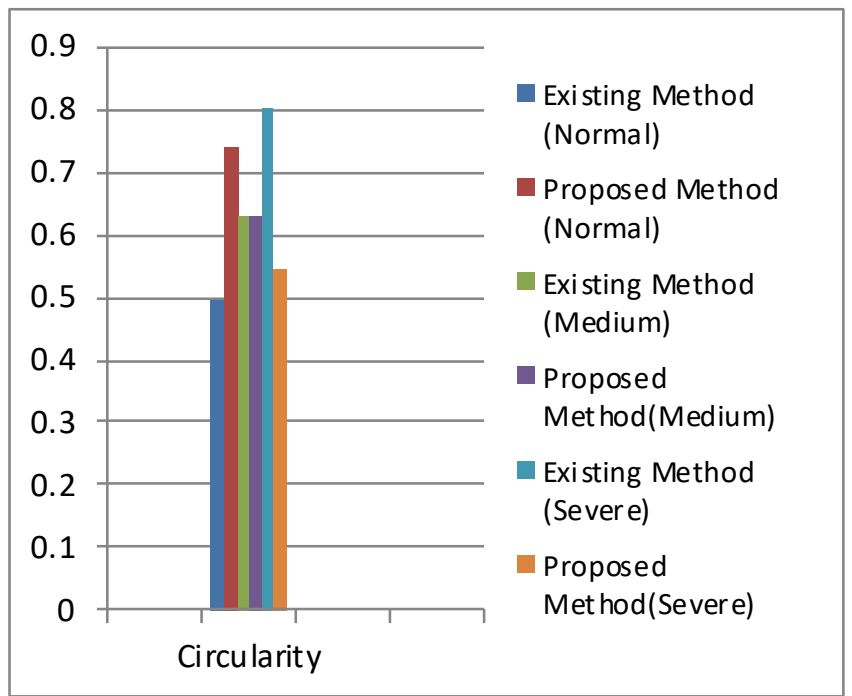

Fig 10: Sum of intensities representation

The sum of all the intensity values represents the total mass of the particular region which is considered for evaluation. As a noticeable and primary means to decide whether a set of digital points has a circular shape, a circularity assessment is projected. Circularity is extensively utilized as a shape measure in computer vision techniques. Circularity quantity is one of the parameters used to formulate a compactness measure in an image for the considered region. A calculation of roundness or circularity (area to perimeter ratio) excluding local inconsistencies can be derived as the ratio of an object's area to a circular area having almost the same convex perimeter. The formula for circularity is expressed as

$$
\mathrm{C}=4 \pi\left(\text { area/ } \text { perimeter }^{2}\right)
$$

A 1.0 value shows a perfect circle. It indicates an increasingly elongated shape as the value approaches 0.0. Values for smaller regions may not be valid. As per the resultant values of circularity and its graphical representation the compactness measure can be investigated for each of the conditions. The measure can be further elaborated that if the ratio is equal to 1 , the object obtained is more or less square or circularly formed. Furthermore, if the ratio reduces from 1, the object is found to be further stretched out. This explanation of circularity can be understood for each method's output of the graphical representation in figure 11 . 


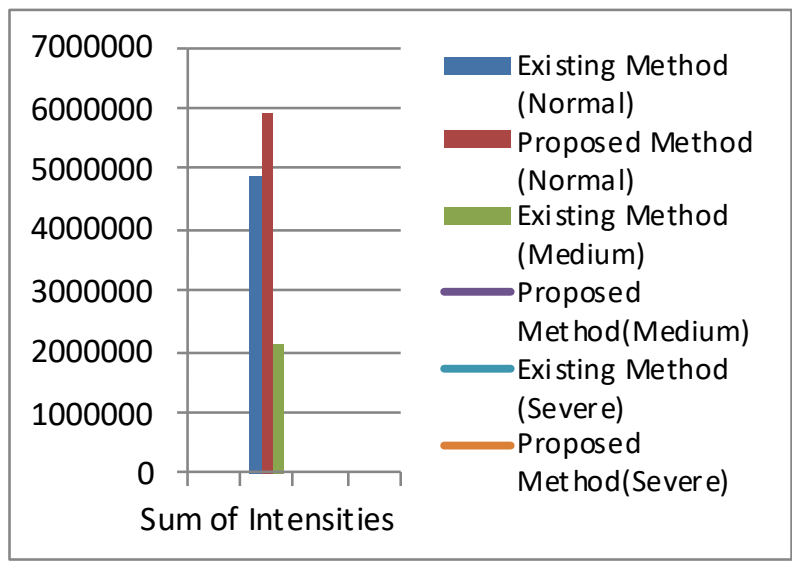

Fig 11: Circularity Representation for both existing and proposed methods

The problem can be clearly understood by scrutinizing the parameters such as area and perimeter and then comparing the input and output images in every form, which are categorized as normal, medium and severe forms. In the similar lines, the tabular values have been represented in the graphical representations for different values in figures $8,9,10$ and 11 . It can be observed that all the parameter values cannot be accommodated in the same graph because of the diversity of the values obtained for each parameter.

\section{Conclusion}

Diabetic Myonecrosis is a rare and complicated issue in Diabetic patients with a history of 5 years of the disease, and it becomes difficult to manage unless proper medication and diet are followed. To aid these patients, we have established a framework which is an automated system through this work. The outcomes provided in this work are mainly focused on the study of irregularities and underlying abnormalities related to Diabetic myonecrosis. The results are analyzed based on both visual perception and using image attributes in a tabular column supported with graphical representations. These results are beneficial to health professionals in creating awareness among diabetic patients and guide patients about how to control diabetes. As a future scope of the work one can continue to be investigating various image processing algorithms and also other abnormalities related to diabetes. Furthermore, a problem that can be considered for research is the relation of joint pains in connection with the proposed application in this paper, as this ailment is mainly found in Type-2 Diabetic patients with lower plasma BDNF (Brain Derived neurotrophic factor) levels which may result in Neurological Disorders.

\section{Acknowledgments}

The authors acknowledge Orthopaedics Journal, Healio, and Applied Radiology Journal and their critical role in the creation of open access available database used in this study and experimentation.

\section{References}

1. Rafael C. Gonzalez, Richard E.woods "Digital Image Processing", Addison-Wesley. An imprint of Pearson Education, 1st edition

2. Sharifi, A., Vosolipour, A., Aliyari Sh, M.; Teshnehlab, M "Hierarchical Takagi-Sugeno Type Fuzzy System for Diabetes Mellitus Forecasting" proc. Of 7th Int. Conf. on Machine Learning and Cybernetics, Kunming, Vol 3, pp.1265 - 1270, 12-15 July 2008 
3. Angervall L, Stener B. "Tumor form focal muscular degeneration in two diabetic patients." Diabetologia1965; 1:39-42 .10.1007/BF01338714

4. Trujillo-Santos AJ. "Diabetic muscle infarction: an under diagnosed complication of long-standing diabetes.” Diabetes Care 2003. Jan;26 (1):211-215 10.2337/diacare.26.1.211

5. Kattapuram TM, Suri R, Rosol MS, Rosenberg AE, Kattapuram SV. Idiopathic and diabetic skeletal muscle necrosis: evaluation by magnetic resonance imaging. Skeletal Radiol 2005. Apr;34(4):203-209 10.1007/s00256-004-0881-8

6. F.A. Peres, F.R. Oliveira, L. A. neves, M. F. Godoy "Automatic Segmentation of Digital Images Applied in Cardiac Medical Images" IEEE -PACHE, Conference, Workshops, and Exhibits Cooperation, Lima, PERU, March 15-19, 2010.

7. Delaney-Sathy LO, Fessell DP, Jacobson JA, Hayes CW. Sonography of diabetic muscle infarction with MR imaging and pathologic correlation. AJR. Am J Roentgen. 2000; 174:165-169.

8. Intajag S, Tipsuwanporn V, Chatthai RC Retinal image enhancement in multi-mode histogram. In: World congress on computer science and information engineering, vol 4, pp 745-749, 2009

9. H. Yue, J. Yang, X. Sun et al., “Contrast enhancement based on intrinsic image decomposition,” IEEE Transactions on Image Processing, vol. 26, no. 8, pp. 3981-3994, 2017.

10. M. Z. Iqbal, A. Ghafoor, and A. M. Siddiqui, "Satellite image resolution enhancement using dual-tree complex wavelet transform and nonlocal means," IEEE Geoscience and Remote Sensing Letters, vol. 10, no. 3, pp. 451-455, 2013.

11. M. M. Riaz, A. Ghafoor, and V. Sreeram, "Fuzzy C-means and principal component analysis based GPR image enhancement," in Proceedings of IEEE International Conference on Radar, pp. 1-4, IEEE, Ottawa, ON, Canada, April 2013.

12. W. Roller, A. Berger, and D. Szentes, "Technology based training for radar image interpreters," in Proceedings of 2013 6th IEEE International Conference on Recent Advances in Space Technologies, pp. 1173-1177, IEEE, Istanbul, Turkey, June 2013.

13. W. Casaca, M. Boaventura, M. P. de Almeida, and L. G. Nonato, "Combining anisotropic diffusion, transport equation and texture synthesis for inpainting textured images," Pattern Recognition Letters, vol. 36, pp. 36-45, 2014.

14. C. C. Chang, B. R. Wu, H. J. Hsu et al., "Texture synthesis approach using cooperative features," in Proceedings of IEEE International Conference on Computer Graphics, Imaging and Visualization, pp. 50-51, IEEE, Sydney, Australia, July 2013.

15. R. C. Gonzalez and R. E. Woods, Digital Image Processing, Pearson, New Delhi, India, 3rd edition, 2009.

16. G. Yadav, S. Maheshwari, A. Agarwal, "Contrast limited adaptive histogram equalization-based enhancement for real time video system", Advances in Computing Communications and Informatics (ICACCI) 2014. Proceedings. International Conference on, pp. 2392-2397, 2014. 
17. Baby Reshma, K.P., Nair, M.S. Multilevel thresholding for image segmentation using Krill Herd Optimization algorithm. Journal of King Saud University - Computer and Information Sciences ,2018.

18. H. Kaut, and R. Singh, A review on image segmentation techniques, Pattern Recognition, vol.26, no.9, pp.1277-1294, 1993.

19. M. Sezgin, and R. Tasaltin, A new dichotomization technique to multilevel thresholding devoted to inspection applications, Pattern Recognition Letters, vol.21, no.2, pp.151-161, 2000.

20. Gui L., Li C., Yang X. "Medical image segmentation based on level set and isoperimetric constraint. Physica Medica: European Journal of Medical Physics. 2017; 42:162-173

21. Chen Y.T. "A novel approach to segmentation and measurement of medical image using level set methods. Magnetic resonance imaging. 2017; 39:175-193.

22. Demirkaya, O, M H Asyali, and P K Sahoo (2008). Image processing with MATLAB: Applications in medicine and biology. CRC Press, Ney York, USA.

23. Bartosz Leszczy'nski et al., Global and Local Thresholding Methods Applied to X-ray Microtomographic Analysis of Metallic Foams, J Nondestruct Eval (2016) 35: 35

24. dos Anjos and H. Shahbazkia, "Bi-Level Image Thresholding-A Fast Method," in Biosignals (2), 2008, pp. $70-76$

25. M. Sezgin and B. Sankur, "Survey over image thresholding techniques and quantitative performance evaluation," Journal of Electronic imaging, vol. 13, pp. 146-166, 2004.

26. N. Otsu, "A threshold selection method from gray-level histograms," IEEE transactions on systems, man, and cybernetics, vol. 9, pp. 62-66, 1979.

27. Mathew J. Mazoch et. Al. "Diabetic Myonecrosis: Likely an Underrecognized Entity" Healio.com, Orthopaedics Journal, October 2014.

28. Parth Desai et. al, "Diabetic Myonecrosis”, Applied Radiology Journal, February 2014.

29. Bardera, I. Boada, M. Feixas, and M. Sbert, Image segmentation using excess entropy, Journal of Signal Processing Systems, vol.54, no.1-3, pp.205-214, 2009.

30. C. Frery, J. Jacobo-Berlles, J. Gambini, and M. E. Mejail, Polarimetric SAR image segmentation with B-splines and a new statistical model, Multidimensional Systems and Signal Processing, vol.21, no.4, pp.319-342, 2010. 